\title{
Zur Lebensituation von Menschen mit Behinderung in Zeiten der Krise
}

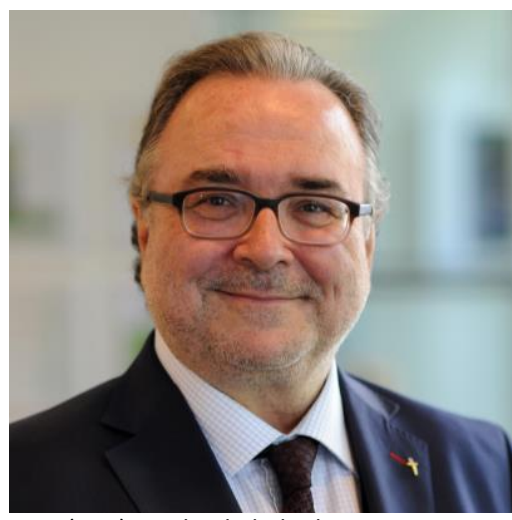

Foto (zvg.): Michael Chalupka
Einer breiten Öffentlichkeit wurde Mag. Michael Chalupka vor allem durch seine vier Amtsperioden als Direktor der Diakonie Österreich und als Bischof der Evangelischen Kirche A.B. bekannt. Er studierte Theologie und war zunächst als Fachinspektor für Religionsunterricht in der Steiermark, Studienleiter am Centro Ecumenico d'Agape in Prali (Italien) und als Pfarrer in Niederösterreich tätig. Ich habe Michael Chalupka als einen Menschen kennengelernt, der sich-jahrzehntelang - einerseits auf sozialpolitischer Ebene für die Lebenssituation von Menschen mit Behinderung einsetzte, andererseits aber auch immer im persönlichen Kontakt bei alltäglichen Herausforderungen und individuellen Problemen zu helfen wusste. Seine medial aufbereiteten Kommentare und öffentlichen Statements übernahmen eine wichtige Rolle in der Behindertenpolitik und waren richtungsweisend für gesellschaftliche Entwicklungen zum Thema Behinderung als Querschnittsmaterie.

Während meiner Zeit im Diakoniewerk in Wien und meiner Arbeit in einer öffentlichen Beratungsstelle für assistierende Technologien lernte ich Hrn. Mag. Chalupka kennen und schätzen. Von ihm stammte eines der einprägsamsten Aussagen in diesem Bereich:

„Nicht der Mensch muss sich an die Technik anpassen, die Technik muss sich dem Menschen anpassen."

Herr Chalupka, Sie waren fast 25 Jahre lang Direktor der Diakonie Österreich und haben einen kundigen Überblick über alle Entwicklungen im Sozial-, Behinderten- und Gesundheitsbereich, welche grundlegend von Bedeutung für die Rechte von Menschen mit Behinderung sind. Wo sehen Sie Österreich im internationalen Vergleich und welche großen Errungenschaften können Sie rückblickend für Menschen mit Behinderung nennen?

Michael Chalupka: Der größte Meilenstein war sicherlich 1993 - noch vor der Ausgliederung von Menschen mit Behinderung aus dem Spitalswesen und psychiatrischen Institutionen und weltweit beispielgebend die Einführung des Pflegegeldes. Es gab erstmals das Denken, eine Leistung für pflegebedürftige und behinderte Menschen zu schaffen und dadurch deren Anspruch auf Unabhängigkeit zu unterstreichen und zu legitimieren. Damit war ein finanzieller Grundstein über Sachleistungen hinausgehend für die 
selbstständige Gestaltung des eigenen Lebens gelegt. Leider wurde durch die Entwertung des Geldes - das Pflegegeld wurde beinahe um ein Drittel seines ursprünglichen Wertes devalviert - im Verlauf der Jahre auch zwangsläufig die Idee dahinter entwertet.

Als eine weitere große Errungenschaft kann ich die Dezentralisierung von großen Einheiten nennen, von großen Wohneinrichtungen hin zu schwerpunktmäßig angelegten Wohngemeinschaften und den individuellen Lösungen, dem voll- oder teilbetreuten Wohnen. Wo ich wenig Entwicklung sehe, ist gegenwärtig die Umsetzung von Diversitätskonzepten, hierbei speziell der gesellschaftlichen sowie innerund nachschulischen Inklusion von Menschen mit Behinderung gemäß dem Richtwert der UNBehindertenrechtskonvention. Hier hat sich im Vergleich zu meiner Tätigkeit als Fachinspektor und Schulamtsleiter vor meiner Zeit in der Diakonie wenig geändert. Die Steiermark hat hier seit der Hochzeit der inklusiven Bemühungen noch immer einen hohen Anteil an Kindern in Inklusionsklassen und in inklusiv ausgerichteten Schulen.

Bewegen wir uns eigentlich weg vom ursprünglichen Auftrag, inklusive Settings aufzubauen und zu unterhalten? Der Bundesentwicklungsplan der allgemeinbildenden Pflichtschulen (vgl. Rahmenzielvorgabe der Sektion Allgemeinbildung des BMBWF u.a.) sieht die „Weiterentwicklung des Lernens und Lehrens an allgemeinbildenden Schulen in Richtung Individualisierung und Kompetenzorientierung" vor und wurde mit dem Zusatz versehen, dass dieses Bemühen „,...in inklusiven Settings“ erweitert betrachtet werden soll. Wie inklusiv sind unsere Schulen?

In vielen Bereichen müssen wir gegenwärtig sogar Rückschritte hinnehmen. Einerseits gibt es in der allgemeinen Inklusionsdebatte einen Stillstand, andererseits muss man festhalten, dass es doch große Unterschiede zwischen den Bundesländern gibt. Diese ebnen sich seit den 80er Jahren landesweit nicht ein und unterliegen über die Jahre hinweg gesehen bei einer großen Schwankungsbreite von ungefähr $25-80 \%$ an inklusiv beschulten Schüler*innen. Hier habe ich in meinem Bereich der evangelischen Schulen deshalb auf Modellversuche, auch in den Gymnasial-formen, gesetzt. Es ist ernüchternd, dass es in manchen Bundesschulen und teilweise vereinzelt in der Oberstufe an allgemeinbildenden höheren Schulen ein mangelndes Bewusstsein für Inklusion gibt. Nicht nur in den Kollegien der Lehrerschaft, auch in der Schulpolitik. Hier bedarf es mehr Aufklärung, Wissen und Akzeptanz, Inklusion nicht nur als Belastung, sondern als Chance zu sehen. Vor allem, wie Schüler*innen mit und ohne Behinderung miteinander lernen können. Lernen an sich kann diverse Formen und Konzepte haben.

\section{Was kann Ihrer Meinung nach bei der Erhöhung der Inklusionsquoten helfen?}

Ein braucht ein breiteres Verständnis für Vielfalt, auch bei einem so großen Thema wie Behinderung. Inklusion kann nicht pauschal abgelehnt werden, es geht letztlich um die Schaffung individueller Rahmenbedingungen zur Integration eines Individuums. Ganz konkret, wenn beispielsweise Menschen mit Autismus-Spektrum-Störung mittels Schulbegleitung, d.h. der Implementierung von Schulassistenz im Regelschulsystem - auch in der Oberstufe - aufgenommen und unterrichtet werden können. Hier ist es wichtig, dass die Schulassistenz kein Modellwesen führt, sondern ein flächendeckender Standard in allen Schulformen wird. Wenn es aber zu keiner Einigung zwischen den Ländern und Ministerien kommt und die Notwendigkeit verkannt wird, Inklusion ganzheitlich zu sehen - auch hinsichtlich der Fördertöpfe und der Zuständigkeiten der Stakeholder*innen der Behörden, dann werden sich einzelne Abteilungen den Ball 
zuspielen und es wird keine Entscheidung getroffen: Weil es letztlich dann immer nur darum geht, eine Budgetlogik auszugleichen. Die lokale Umsetzung ist ohne rechtlichen Anspruch und ohne Grundlage oft schwierig.

\section{„Je privilegierter man lebt, desto einfacher war die Bewältigung der Situation."}

Wie würden Sie die Situation von Menschen mit Behinderung $(\mathrm{MmB})$ in den vergangenen zwölf Monaten der COVID-19-Pandemie beurteilen? Kann man abschätzen, welche Auswirkungen die pandemiebedingten Maßnahmen auf die Lebenssituation von Menschen mit einer Behinderung hatten, etwa hinsichtlich Isolation, Teilhabe am öffentlichen Leben, Umsetzung der Maßnahmen, Risikogruppenzugehörigkeit" usw.?

Die COVID-19-Pandemie hat insgesamt eines deutlich gezeigt: Je privilegierter man lebt, desto einfacher war die Bewältigung der Situation. Für Menschen mit finanziellen Sorgen, auch für Familien mit beschränktem Wohnraum, sind die Krise und die Belastungen dadurch dramatischer. Menschen mit Behinderung hat es noch einmal viel schwerer getroffen: Viele Unterstützungssysteme sind, wenn auch nur kurzfristig, weggefallen, die soziale und gesellschaftliche Teilhabe war erneut ein großes Problem. Je nach Art der Beeinträchtigung und dem Grad der Behinderung hat es sich anders ausgewirkt, aber grundsätzlich ist es notwendig, dass eine Kontinuität, eine Routine sowie eingespielte Abläufe beibehalten werden, wenn Teile des öffentlichen wie privaten Lebens wegbrechen.

Inwiefern hat dies auch Einrichtungen und Institutionen betroffen, etwa Kindergärten, Schulen, Wohneinrichtungen, Tages- und Werkstätten, Pflegeeinrichtungen usw.?

Durch die wechselnden Vorschriften und die sich rasch verändernden Realitäten wurde die Aufrechterhaltung von Normalität und damit der Stabilität eine Herausforderung. Die Isolation und die Besuchsbeschränkungen in den Pflegeheimen und in der Altenfachbetreuung waren zum Beispiel ein langanhaltendes Problem und eine große Belastung.

Diese Ausgabe von \#schule_verantworten heißt „Führung in Zeiten der Krise“ - Sie waren und sind bereits mehr als ein Vierteljahrhundert als Geschäftsführer und Führungskraft tätig und hatten mehrere Entwicklungsbereiche und Initiativen zu verantworten. Was können Sie in der aktuellen Pandemie Menschen, die in einer Führungsposition und auch bildungsverantwortlich sind, in Hinblick auf eine gelingende Führungskultur in Krisenzeiten mit auf den Weg geben?

Im Wesentlichen: Es ist bedeutsam, kontinuierlich, klar und rasch zu informieren, die Kommunikation zu suchen sowie Stabilität und Sicherheit zu vermitteln. Auf allen Ebenen gab es essentielle Verunsicherungen und große Ängste, es ist eine Zeit der Unvorhersehbarkeit. Unsere Angewohnheit Pläne zu machen, volle Terminkalender abzuarbeiten, Projekte zu verfolgen und Meilensteine anzustreben ist weggefallen. Es bedarf daher einer unmittelbaren Klarheit und Transparenz in der Führung, man muss auch die 
Unverfügbarkeit annehmen und akzeptieren, dass gegenwärtig nicht alles machbar, gestaltbar und umsetzbar ist. Viel in der Krisenkommunikation der zuständigen Stellen und der Regierung war mehr Public Relations und eine Erzählung, bestenfalls ein Richtwert für die Praxis.

Der Wunsch nach klaren Entscheidungen und einer Eindeutigkeit war bei allen, die eine Einrichtung leiten müssen, vorhanden. Eine klare Kommunikation heißt aber nicht zwangsläufig, dass es keine Widersprüche gibt. Die Situation im Moment ist ja an sich oft widersprüchlich.

\section{Das heißt, wir hatten mit Problemen zu tun, für deren Lösung uns die Referenzwerte fehlten?}

Zum einen sicherlich, aber die Corona-Krise hat nicht alle Probleme geschaffen (zum Beispiel sind in Zeiten des Social Distancings ausgrenzungsgefährdete Personengruppen noch stärker betroffen) - genauso problematisch ist, dass die Pandemie wie ein Vergrößerungsglas für Probleme wirkt, die es auch vorher schon gegeben hat.

\section{Ein Brandbeschleuniger für soziale Problemfelder?}

Genau, aber das zeigt sich auch in gewissen Bereichen des öffentlichen Lebens und der allgemeinen Kommunikation. Wenn es beispielsweise noch nicht selbstverständlich ist, Entscheidungen und Mitteilungen der Regierung und der Behörden für Menschen mit einer intellektuellen Behinderung oder kognitiven Einschränkung in einfacher Sprache und den Richtlinien für leichter Lesen mitzuteilen, dann wird das in Krisenzeiten auch nicht passieren - dann ist es zu spät, das hätte schon vorher, im "Normalzustand“ umgesetzt werden müssen. Dort, wo es Lücken gibt, sind sie auch in der Krise vorhanden - unter Umständen sogar deutlicher. In der Krise kann man keine Regelkommunikation oder Automatismen etablieren, wenn dies vorher verabsäumt wurde.

Sie haben über Jahrzehnte die bildungspolitischen Entwicklungen im Sozial-, Schul- und Behindertenbereich gestaltet, beobachtet und kommentiert. Welche Weichenstellungen in der Politik haben MmB nachtäglich besonders geholfen - welchen Bedarf sehen Sie in der Gegenwart, bzw. was wünschen Sie sich für die Zukunft?

Was noch immer nicht umgesetzt wurde, ist der Rechtsanspruch auf bestimmte assistierende Technologien für Menschen mit Behinderung, vornehmlich Kommunikationshilfsmittel für Menschen mit keiner oder einer eingeschränkten Lautsprache. Während eine Arbeitsplatzadaption im Rahmen der beruflichen Integration gefördert wird, sind die Gesetzeslage und der Rechtsanspruch auf reine Kommunikationshilfsmittel in Österreich und der EU sehr unterschiedlich geregelt. Es ist schade, dass der Weg zur Finanzierung für die Betroffenen noch immer ein Hürdenlauf ist. Der Anspruch auf eine vollständige Kostenübernahme von Hilfsmitteln steht jetzt mittlerweile im dritten Regierungsprogramm. Dass sich Menschen, die in der Kommunikation beeinträchtigt sind, noch immer als Bittsteller*innen fühlen müssen und auf Förderungen und private Fundraising-Kampagnen angewiesen sind, ist in meinen Augen mehr als unwürdig.

\section{Haben Sie ein abschließendes Statement für den Schulbereich?}

Ich glaube, ich habe (für meinen Bereich) die Polarisierung rund um die „bessere" Schulform oder, besser gesagt die „geeignetere“ Unterrichtsform nie richtig nachvollziehen können. Es geht doch letztlich darum, 
so viele Schüler*innen mit einer Behinderung wie möglich an einem individuell zugeschnittenen Bildungsprozess im Regelschulsystem teilhaben zu lassen. Das heißt im Umkehrschluss nicht, dass es deswegen weniger Fördermaßnahmen im Regelschulsystem geben muss oder parallel dazu keine spezialisierten Sonderschulstandorte geben kann. Aber man darf aufgrund einer kleinen Personengruppe, die eine sehr individuelle Lösung benötigt, nicht einem Großteil der anderen Kinder mit einer Behinderung das Recht auf Inklusion verwehren.

Vielen Dank für das Gespräch!

Mit Michael Chalupka sprach Matthias Hesse 